\title{
Malassezia Species Associated With Dermatitis in Dogs and Their Antifungal Susceptibility
}

\author{
Uday Seetha, Sumanth Kumar, Raghavan Madhusoodanan Pillai, \\ Mouttou Vivek Srinivas", Prabhakar Xavier Antony and Hirak Kumar Mukhopadhyay
}

Department of Veterinary Microbiology, Rajiv Gandhi Institute of Veterinary Education and Research, Pondicherry-605 009, India

*Corresponding author

\begin{tabular}{|l|}
\hline K e y w o r d s \\
$\begin{array}{l}\text { Malassezia pachydermatis, } \\
\text { Sabouraud's dextrose agar, } \\
\text { Modified Dixon's agar, } \\
\text { Polymerase chain reaction, } \\
\text { Antifungal Susceptibility } \\
\text { testing }\end{array}$ \\
\hline Article Info \\
\hline $\begin{array}{l}\text { Accepted: } \\
\text { 18 May 2018 } \\
\text { Available Online: } \\
\text { 10 June } 2018\end{array}$ \\
\hline
\end{tabular}

\section{A B S T R A C T}

The present study was taken with the objective of isolation, characterization, molecular detection and antifungal sensitivity of Malassezia species from dermatitis cases from dogs in and around Pondicherry state. A total of 100 skin swabs were collected from the dogs showing dermatological problems suggestive of Malassezia. Out of 100 swabs, 41 Malassezia isolates were successfully isolated and had good growth on Sabouraud's Dextrose Agar (SDA) during the primary isolation from the skin swabs. Biochemical tests for catalase, $\beta$-glucosidase activities and the capability to grow with three water soluble lipid supplements, namely Tween 20, Tween 80 and Cremophor EL concluded that the $M$. pachydermatis was the sole species isolated from the cases of canine dermatitis in Pondicherry state. Cytological examination revealed that direct skin swab smear was more sensitive than adhesive tape technique and impression smear. The frequency of isolation of $M$. pachydermatis was higher in neck region (8) followed by other regions in canine. Out of 100 skin swabs screened using an M. pachydermatis species specific primers, 61 were identified positive. The study showed a higher sensitivity of PCR (61\%) in detecting Malassezia dermatitis over culture (41\%). Based on invitro antifungal susceptibility studies it can be concluded that Ketoconazole, Itraconazole, Fluconazole and Amphotericin B can be advocated as the drug of choice to treat Malassezia dermatitis in this geographical location.

\section{Introduction}

Malassezia has been classified as dimorphic fungi since it has been found to exist in both yeast and mycelial phases.

The yeast form was named as Pityrosporum and the mycelial form as Malassezia. However, in 1986 they were recognized to be two forms of the same organism and were thus collectively named Malassezia (Cannon, 1986). Malassezia considered as a normal cutaneous inhabitant of skin in humans, animals and birds. These lipophilic fungi colonize in the stratum corneum layer of skin, which is rich in lipids. These Dimorphic lipophilic fungi sometimes act as opportunistic pathogens and cause the dermatological infections such as seborrhoeic dermatitis and otitis in domestic animals. 
The Malassezia yeast cells are round, oval or cylindrical and may vary in size from 1 to $8 \mu \mathrm{m}$ diameter (Keddie, 1966). They reproduce asexually by budding from a broad base (Chen and Hill, 2005). The cell wall of Malassezia is very thick $(0.12 \mu \mathrm{m})$ and multilayered, consisting mainly of sugars $(70 \%)$, lipids (15$20 \%$ ) and proteins (10\%) (Ashbee and Evans, 2002). All Malassezia species except Malassezia pachydermatis requires an exogenous source of long chain fatty acids like $\mathrm{C} 14$ or $\mathrm{C} 16$ fatty acids for their growth.

The genus Malassezia belongs to the Phylum Basidiomycota, comprises of 14 species, which have been identified based on their morphology, biochemical features and molecular analysis. Initially, seven species $(M$. furfur, M. obtusa, M. globosa, M. slooffiae, $M$. sympodialis, $M$. pachydermatis and $M$. restricta) were described (Gueho et al., 1996). Later, seven new Malassezia spp. (M. dermatis, M. equina, M. japonica, M. nana, M. yamatoensis, M. caprae and M. cunicoli) were identified using biochemical, morphological, biological and molecular analysis (Sugita et al., 2004; Hirai et al., 2004; Cabanes et al., 2007).

M. pachydermatis produces proteolytic enzymes that can damage the epithelium leading to hyperplasia with enlargement of the ceruminous glands (Nicklas and Mumme, 1979). Malassezia produces keratinase and other enzymes capable of digesting the keratin protein complex, allowing the organism to burrow deeper into the stratum corneum in the host and elicit an inflammatory reaction (Guillot, 1995).

The lesions are usually first seen on the abdominal skin, but may spread to the entire abdomen, the axilla and the inguinal region (Larsson et al., 1988). In diseased ears, however, $M$. pachydermatis can be readily found alone or associated with bacteria, mainly Staphylococcus spp. M. pachydermatis can also be isolated from the ears of clinically healthy animals (Mansfield et al., 1990).

Histopathologically, the lesions are characterized by inflammation, perivascular infiltration, epidermal and follicular hyperkeratosis with many budding yeast cells in the stratum corneum (Mason and Evans, 1991). The organism is not restricted to dogs alone, as it has been isolated from several other mammalian species and birds (Scott, 1992). Zoonotic transfer has been documented from dogs to immunocompromised patients by health care workers who own dogs (Chang et al., 1998).

Dermatitis due to Malassezia species in canines is recognized with increasing frequency in veterinary practice in Pondicherry state (Southern India). Since no systematic investigations were carried out with regards to the occurrence and treatment of Malassezia infections in this region, the present study was aimed with the Isolation, characterization followed by PCR-based detection and Antifungal sensitivity of Malassezia species from dermatitis dogs cases in and around Pondicherry state.

\section{Materials and Methods}

\section{Sample collection and Processing}

A total of 100 skin swabs were sampled from the dog cases came to the Teaching Veterinary Clinical Complex, RIVER, Pondicherry (33 nos) and Animal husbandry department, Pondicherry (67 nos) showing dermatological problems suggestive of Malassezia dermatitis like erythema, hyperpigmentation, greasy exudates, scaling etc (Supplementary Figure $1)$.

Sterile cotton swabs moistened with sterile distilled water were used to collect the 
specimen. The cotton swab was rolled and rubbed firmly against the entire skin area for 10 seconds from the dogs showing dermatological problems suggestive of Malassezia dermatitis. The swab was then transferred onto the sterile test tube and transported to laboratory within 2 to 4 hours of the collection.

\section{Cytological studies}

\section{Impression smear examination}

The impression Smears were made in a clean glass slide directly from the surface of the lesion. Then the heat fixed impression smears were stained with Methylene blue stain to detect the presence of Malassezia yeasts under oil immersion objective. More than one yeast cell per field is considered positive for Malassezia dermatitis (Nardoni et al., 2008).

\section{Adhesive tape examination (Scotch test)}

Here the impression is made with the adhesive surface of the cellophane tape placed onto the affected skin surface firmly for $2-3 \mathrm{sec}$ and then sticked onto a clean microscopic slide containing 2-3 drops of lactophenol cotton blue (LCB) mount. The tape strip stained with LCB mount for 2 minutes was examined under high power objective microscope to detect the presence of Malassezia yeasts.

\section{Skin swab smear}

The smears are made by rolling / rubbing the wet cotton swab firmly against the entire skin lesions area for 10 seconds and then rolled / rubbed over the microscopic glass slide. Then the heat fixed impression smears were stained with Methylene blue stain to detect the presence of Malassezia yeasts under oil immersion objective. More than one yeast cell per field is considered positive for Malassezia dermatitis (Nardoni et al., 2008).

\section{Isolation and identification}

The skin swabs were plated on Sabouraud's dextrose agar (SDA) and Modified Dixon's agar (MDA) and incubated at $37^{\circ} \mathrm{C}$ for 7 days for the isolation of Malassezia yeasts. The colonies showing cultural characters of Malassezia were examined microscopically for the typical morphology and were further subjected for a) Catalase activity, b) $\beta$ glucosidase test, c) Tween and Cremophor EL utilization biochemical tests to identify them upto species level (Cafarchia et al., 2011).

\section{Catalase activity}

A loopfull of test culture on SDA agar was mixed with 2-3 drops of 3 per cent $\mathrm{H} 2 \mathrm{O} 2$ on a clean glass slide and examined for the release of nascent oxygen in the form of gas bubbles. A positive reaction was indicated by the effervescence of oxygen within 1-2 min. (Gueho et al., 1996).

\section{$\beta$ glucosidase test}

$\beta$ glucosidase test was done by inoculating a loop full of fresh culture into esculin iron agar medium and incubated at $37^{\circ} \mathrm{C}$ for 5 days. A positive reaction was indicated by the blackening of the medium which reveals that Malassezia organism possess a $\beta$ glucosidase that is able to hydrolyse the glucosidic bond of esculin, thus liberating glucose and esculin. The phenol moiety reacts with the iron to give a black colour (Kaneko et al., 2006).

\section{Tween and Cremophor EL utilization}

Two loops of a 4 to 5 day old Malassezia culture were suspended in $3.0 \mathrm{ml}$ of sterile demineralised water. This inoculum was added to molten SDA maintained at $50^{\circ} \mathrm{C}$, and the mixture was poured immediately in a $9 \mathrm{~cm}$ petri dish. After complete solidification, wells were made with a $2 \mathrm{~mm}$ punch, devoted to test 
the growth using Tween 20, Tween 80 and Cremaphor EL. The wells are filled with approximately $15 \mu \mathrm{l}$ of each product and petri dishes are incubated for 7 to 10 days at $37^{\circ} \mathrm{C}$ in a moist environment, and turned upside down on the second day to delay their dehydration. A positive reaction was indicated by presence of dense growth around the wells in a disk like pattern.

\section{Antifungal susceptibility test}

Antifungal susceptibility test was carried out as per the standard disc diffusion method described by Bauer et al., (1966). Sabouraud's dextrose agar ( $\mathrm{pH} 5.6 \pm 0.2)$ was employed for antifungal susceptibility test. Three to five pure individual colonies were inoculated into $3 \mathrm{ml}$ of $0.04 \%$ Tween 80 and incubated at $37^{\circ} \mathrm{C}$ for $2 \mathrm{hr}$. Then the lawn culture was made with the swab from the incubated inoculums onto the SDA plates. After lawn culture was made the SDA plates were placed in the refrigerator for $2 \mathrm{hr}$ for better absorption of the culture. Following antifungal discs with the mentioned concentrations were applied over the plated SDA. Itraconazole (It) $10 \mu \mathrm{g}$, Ketoconazole (Kt) $10 \mu \mathrm{g}$, Nystatin (Ns) 100 I.U, Fluconazole $(\mathrm{Fu}) 10 \mu \mathrm{g}$, Amphotericin B

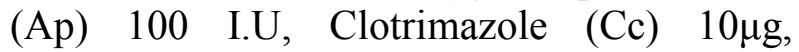

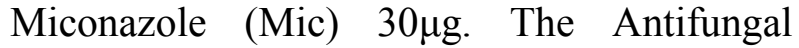
impregnated plates were incubated in the inverted position, at $37^{\circ} \mathrm{C}$ for $48 \mathrm{hr}$. The interpretation of zone diameter was carried out according to the standards laid down by Clinical Laboratory Standards Institute (CLSI), formerly known as National Committee for Clinical Laboratory Standards (NCCLS). The diameter of zone of inhibition was translated into sensitive or resistance.

\section{Polymerase Chain Reaction}

Boiling-lysis method of extraction of DNA from the clinical sample and culture recommended by Zhang et al., (2004) with slight modifications was followed. The clinical sample / culture was boiled in a water bath at $96^{\circ} \mathrm{C}$ for $10 \mathrm{~min}$ followed by snap chilling for 5 mins and centrifugtion at $16,000 \mathrm{~g}$ for $5 \mathrm{~min}$. The supernatant was used as a template DNA in a PCR reaction.

The PCR amplification was carried out with the species specific primer pair M.pa $a_{\text {for }} \&$ $\mathrm{M}^{\mathrm{ppa}} \mathrm{a}_{\mathrm{rev}}$ with the Initial denaturation at $94^{\circ} \mathrm{C}$ for $3 \mathrm{~min}$, followed by 30 cycles at final denaturation at $94^{\circ} \mathrm{C}$ for $30 \mathrm{~s}$, annealing at $62^{\circ} \mathrm{C}$ for $1 \mathrm{~min}$, extension at $72^{\circ} \mathrm{C}$ for $40 \mathrm{~s}$ and a final extension at $72^{\circ} \mathrm{C}$ for $10 \mathrm{~min}$. Following PCR, the amplified product was analyzed by submariane gel electrophoresis electrophoresis

\section{Results and Discussion}

\section{Isolation and identification}

Samples collected from 100 dogs with symptoms suggestive of Malassezia dermatitis were incubated at $37^{\circ} \mathrm{C}$ for 7 days on SDA and 15 days on MDA. Growth of Malassezia was observed from 4 to 7 days on SDA whereas on MDA the growth was observed from 9 to 15 days. Based on this result, it could be concluded that SDA is a preferable medium for the isolation of Malassezia pachydermatis compared to MDA although many workers have suggested the superiority of Modified Dixon's agar for the isolation of lipid dependent species of Malassezia from canine dermatitis (Nardoni et al., 2006, Galuppi et al., 2010 and Cafarchia et al., 2011).

Out of 100 samples collected, 41 isolates of Malassezia spp. were isolated successfully. The colonies of Malassezia spp. were macroscopically visible over 3-5 days when incubated at a temperature of $37^{\circ} \mathrm{C}$ whereas; the growth was weak when incubated at room temperature $\left(25^{\circ} \mathrm{C}\right)$. The colonies were raised or high convex and smooth with cream colour 
initially (Figure 1) and later became dry, wrinkled and orange to brown in colour (No shown). On microscopic examination of the isolated colonies using Grams staining procedure; the organisms appeared dark blue colored with unique peanut or footprint shaped (Figure 2).

\section{Biochemical tests for Malassezia species}

The identification of Malassezia yeasts is done by using specific biochemical tests like catalase test, $\beta$ glucosidase activities and the capability to grow with three water soluble lipid supplements, namely Tween 20, Tween 80 and Cremophor EL. In this research, all the 41 Malassezia yeast isolates had a very slow or weak activity for catalase. These results are similar to those of Galuppi et al., (2010) who have reported very weak catalase activity in 23 strains of $M$. pachydermatis. Similarly, all the 41 Malassezia yeast isolates were also positive for $\beta$ glucosidase activity. 41 Malassezia isolates utilized Tween 20, Tween 80 and Cremophor EL by showing good growth around them (Figure 3). These results were similar to Galuppi et al., (2010) who recorded good growth around the wells of Tween 20, Tween 80 and Cremophor EL. Based on the above tests in isolates, the study concludes that $M$. pachydermatis was the sole species isolated from the cases of canine dermatitis. In this work, the lack of recovery of lipid dependent species could be ascribed to the strongly modified skin habitat due to atopy. These alterations could have determined an overgrowth of $M$. pachydermatis with the consequent inhibition of lipid dependent species, less adapted to the cutaneous microhabitat of dogs.

\section{Polymerase chain reaction}

Out of 100 canine dermatitis clinical samples (skin swabs from the lesions) screened using an $M$. pachydermatis species specific primers
(Table 1) derived from the internal transcribed spacer region of the rRNA gene, 61 were found positive with the expected product size of $220 \mathrm{bp}$ (Figure 4). Culture evidence indicated that only $41 \%$ of the cases were positive whereas $61 \%$ of cases were positive for Malassezia infection by PCR assay. Chi square analysis also revealed significant differences $(\mathrm{P}<0.5)$ between isolation and PCR assay in the diagnosis of Malassezia pachydermatis.

Among the 100 infected animals included in this study, the highest occurrence was noticed in dogs of one to three years of age $(56 \%)$ followed by six months to one year of age $(19 \%)$, three to six years of age $(16 \%)$, zero to six months of age (6\%) and above six years of age (3\%). Age wise occurrence of Malassezia infections is depicted in Table 2. This is in concurrence with the earlier reports of Girao et al., (2006) who opined that majority of dogs with $M$. pachydermatis were aged between one and three years of age.

The study showed that Malassezia dermatitis was markedly prominent in Non- descriptive dogs $(55 \%)$ followed by Labrador Retriever and Spitz (13\%), German Shepherd (11\%) Chinese Pug (4\%), Dalmatian (2\%), Doberman and Great Dane (1\%). The detailed breed wise occurrence of Malassezia dermatitis is presented in Table 3. Certain breeds like American cocker spaniel, Springer spaniel, Basset Hound, Daschund, English setter, West Highland terrier, Silky terrier, German Shepherd, Poodles, Chihuahua and Collie are reported to be at increased risk (Plant et al., 1992, Bond et al., 1996, Maulldin et al., 1997, Charach 1997 and Muller et al., 2001). In Puducherry region, most of the middle and low income groups of people were rearing Non descriptive breeds of dogs due to their economic status. This might have influenced the highest percentage of incidence of disease among Non-descriptive dogs. 
Fig.1 Growth of $M$, pachydermatis was observed on an average of 4 to 7 days on SDA which were incubated at $37^{\circ} \mathrm{C}$. The colonies were raised, high convex, smooth with cream colour initially and later (not shown) became dry, wrinkled and orange to brown in colour

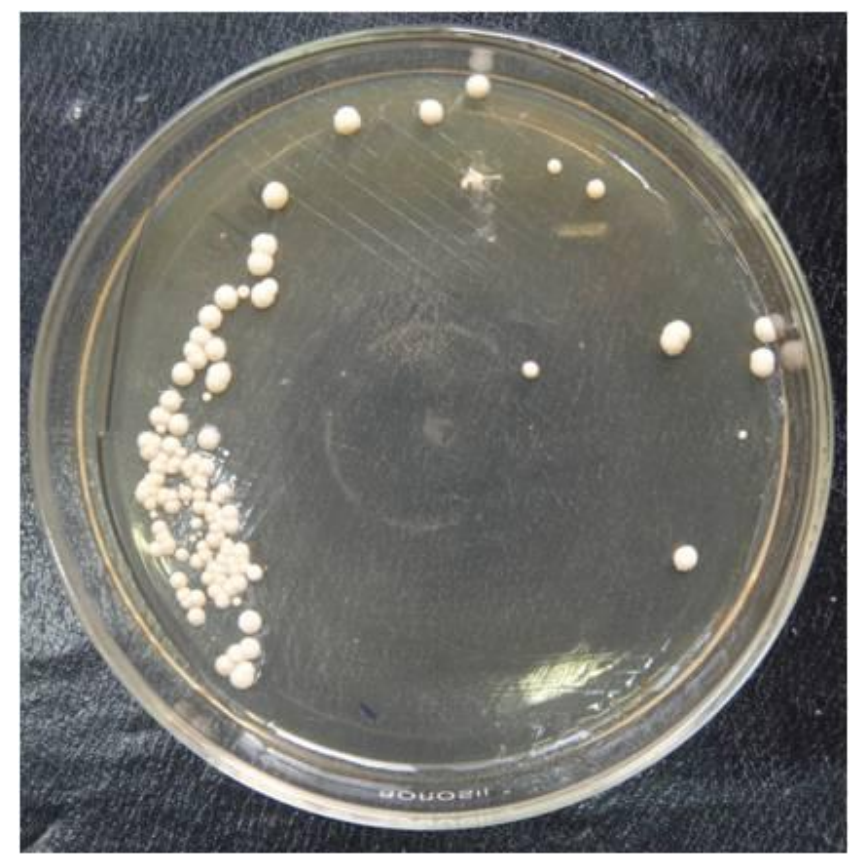

Fig.2 Skin swab cultured isolate showing the microscopic morphology (1000X magnification). Peanut or footprint shaped budding yeast cells of $M$. pachydermati demonstrated by Methylene blue staining technique
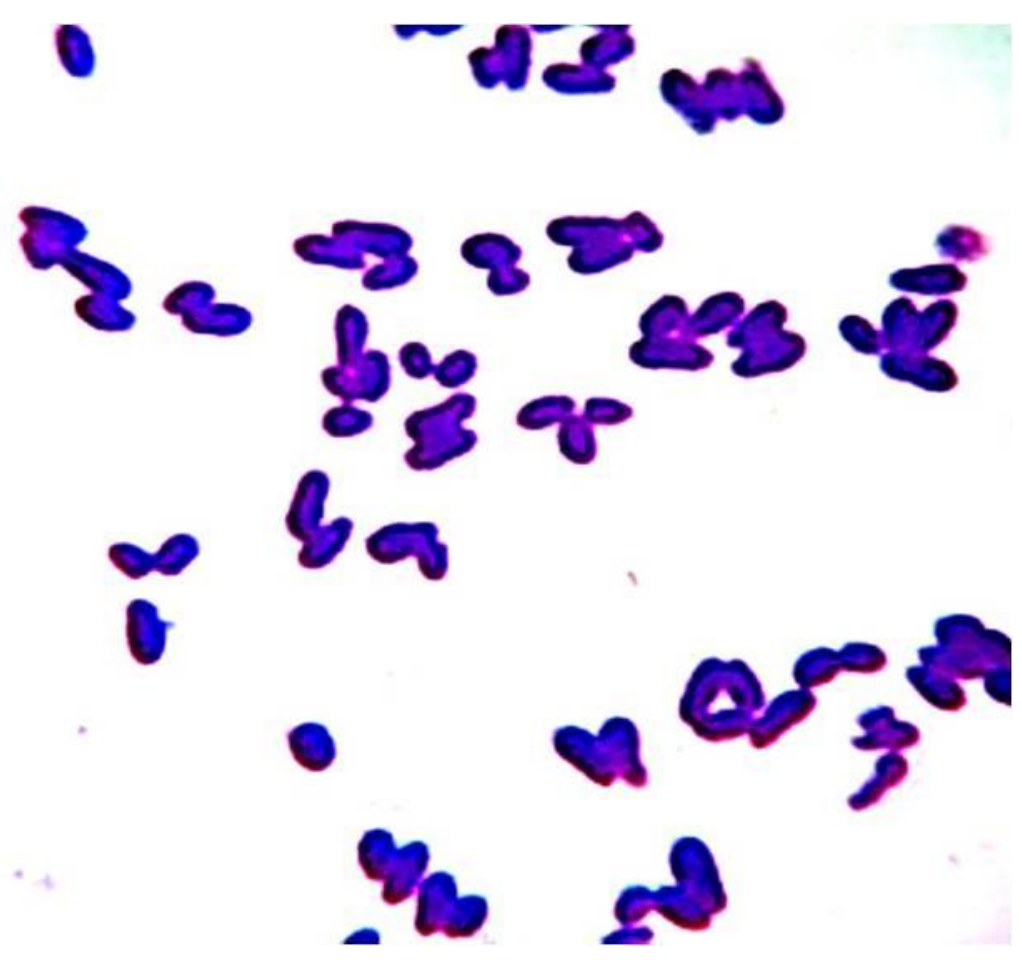
Fig.3 Utilization of Tween 20, Tween 80 and Cremophor EL by Malassezia isolate; showing good growth around them. 1. Tween 80, 2. Cremaphor EL, 3. Tween 20

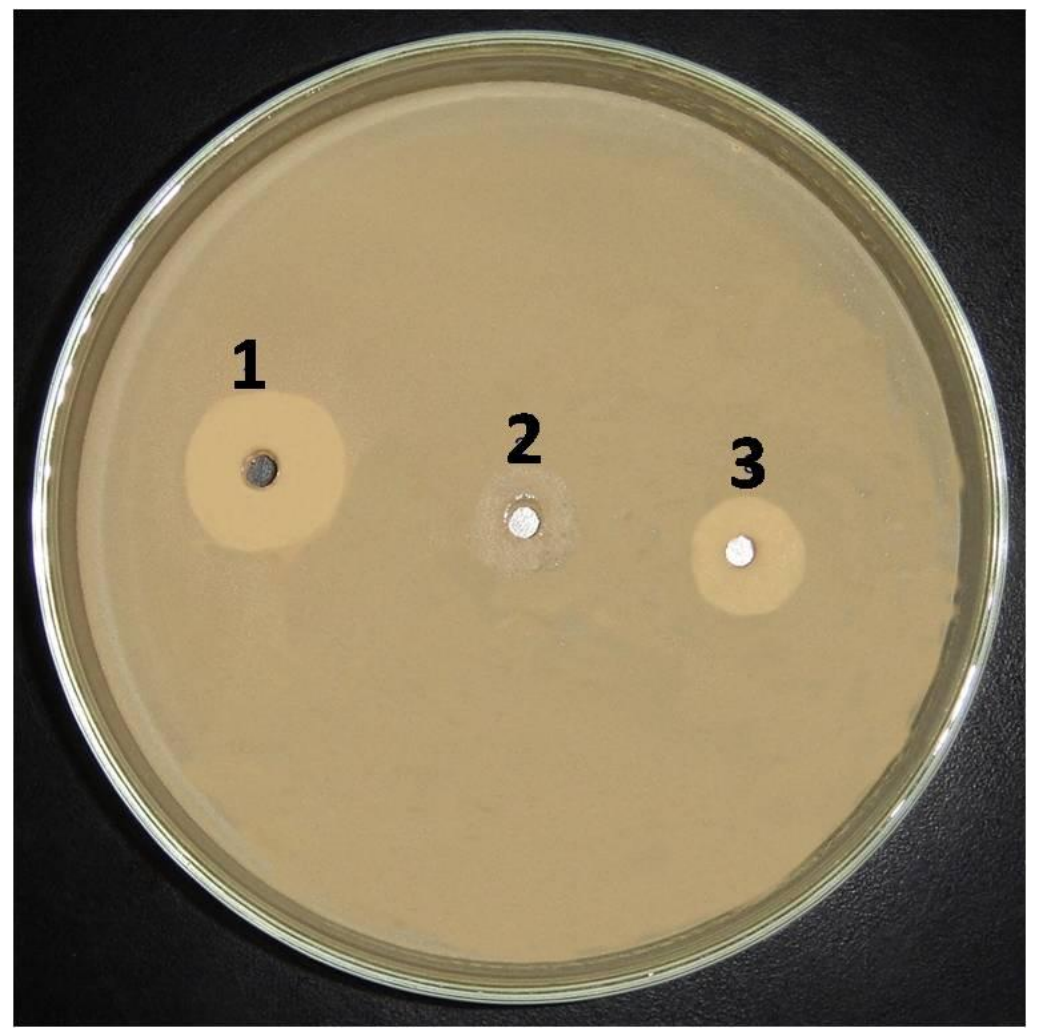

Fig.4 Screening of Clinical swabs / isolates for Malazzesia pachydermatis by PCR amplification using M.pa $a_{\text {for }} \&$ M.pa $_{\text {rev }}$ primer pair; Lane $1 \&$ 2- Clinical samples Lane $3 \& 4$ - isolates, Lane 5 - Negative control, Lane 6 - Positive control \& Lane 7 - DNA Marker

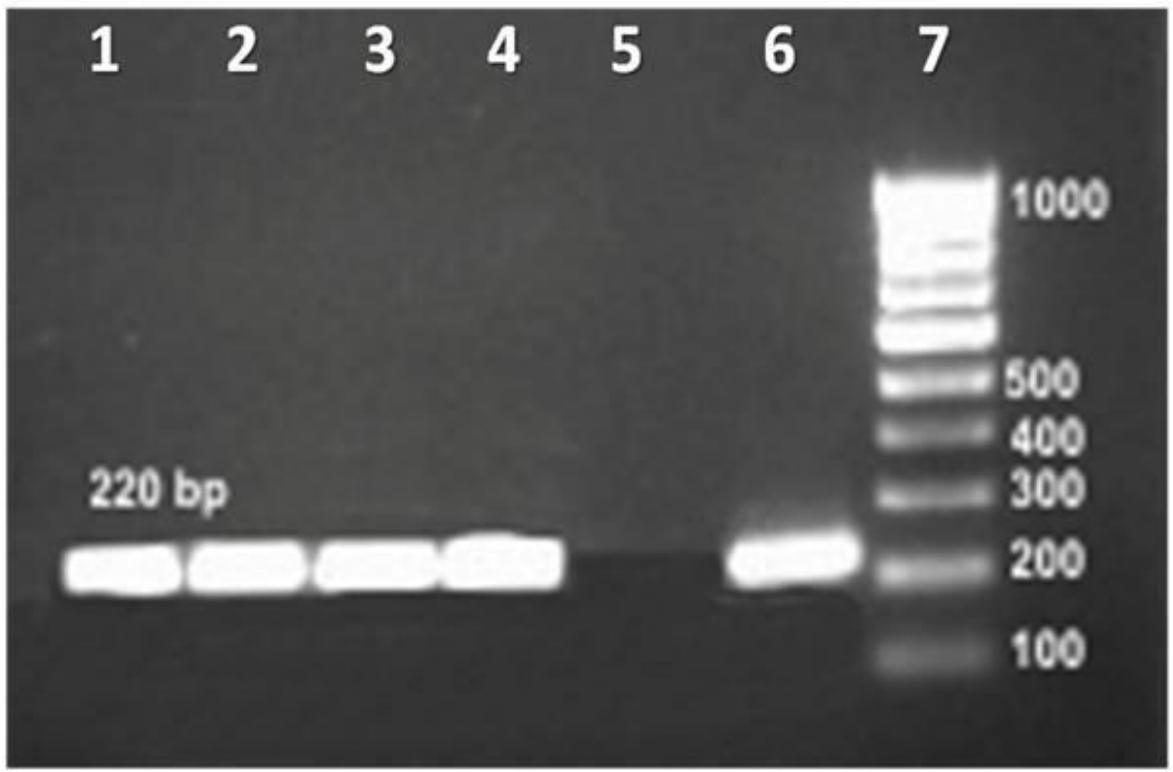


Fig.5 Antifungal susceptibility test carried out with seven antifungal agents against one of the clinical isolate. The isolate shows that it is sensitive to Amphotericin B (A), Ketoconazole (B), Itraconazole (C), Fluconazole (D), Miconazole (E) and Clotrimazole (F) but resistant to Nystatin (G)

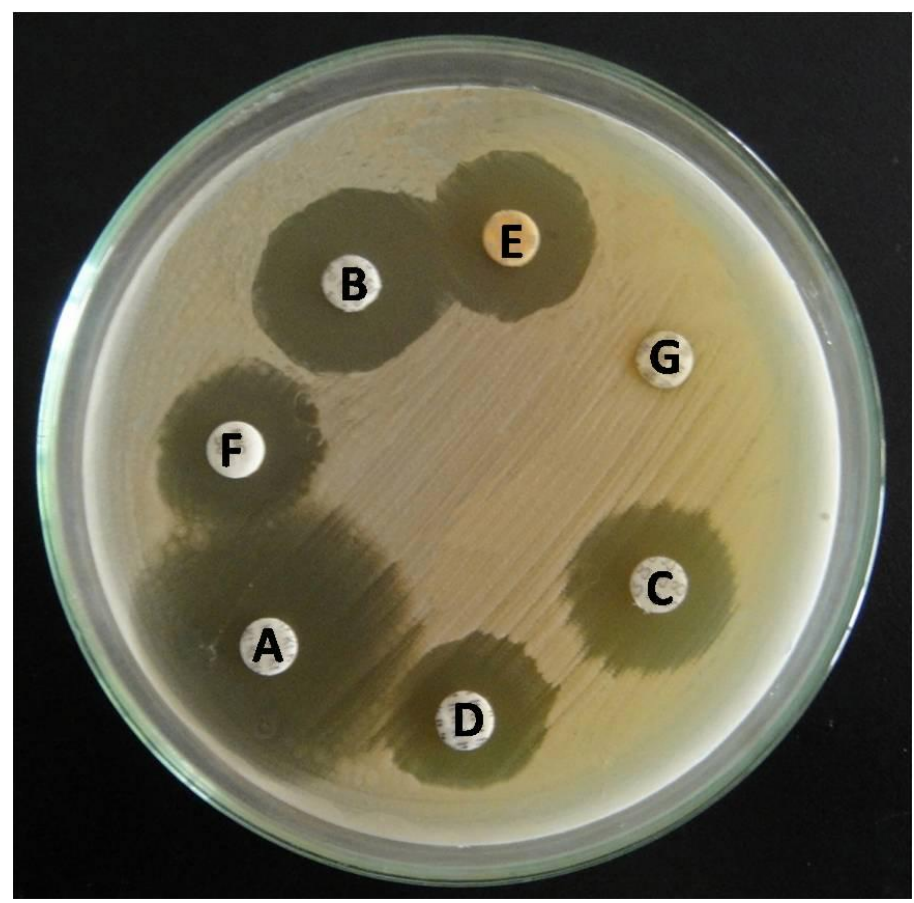

Supplementary Fig.1 The dog cases came to the Teaching Veterinary Clinical Complex, RIVER, Pondicherry showing dermatological problems such as A) Scaling, B) Otitis externa, C) Hyperpigmentation and D) Erythema, etc suggestive of Malassezia dermatitis

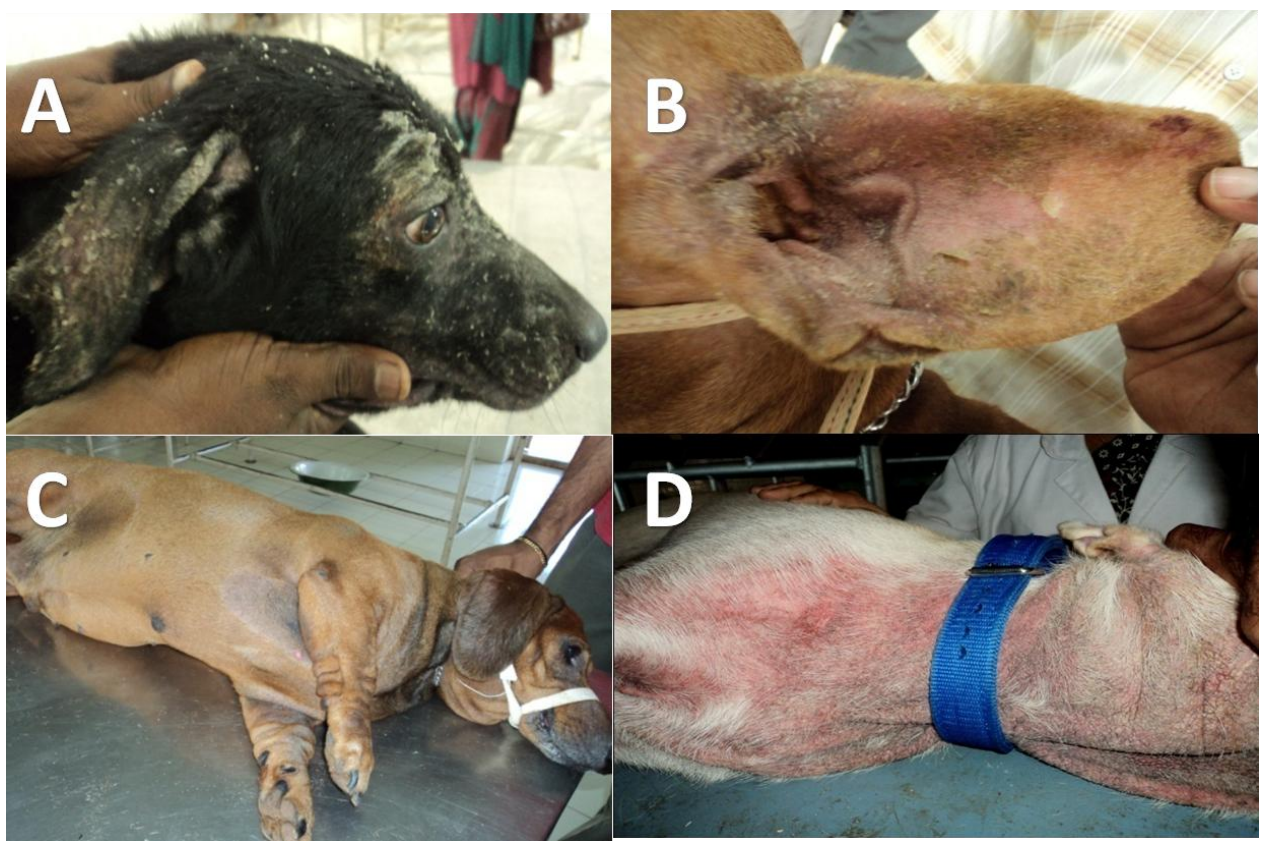


Table.1 Oligonucleotide primers used for amplification for Malassezia pachydermatis gene

\begin{tabular}{|l|l|l|l|}
\hline Target & Primers & Sequence $\left(\mathbf{5}^{\prime} \rightarrow \mathbf{3}^{\prime}\right.$ ' direction) & $\begin{array}{l}\text { Amplicon size } \\
(\mathbf{b p})\end{array}$ \\
\hline $\begin{array}{l}\text { Malassezia } \\
\text { pachydermatis }\end{array}$ & $\mathbf{M . p a}_{\text {for }}$ & CTGCCATACGGATGCGCAAG & 220 \\
\hline $\mathbf{M . p a}_{\text {rev }}$ & TTCGCTGCGTTCTTCATCGA & \\
\hline
\end{tabular}

Table.2 Age wise occurrence of Malassezia dermatitis in dogs

\begin{tabular}{|l|}
\hline AGE \\
\hline $\mathbf{0}$ to 6 months \\
\hline $\mathbf{6}$ months to 1year \\
\hline $\mathbf{1}$ to 3 years \\
\hline $\mathbf{3}$ to 6 years \\
\hline > 6 years \\
\hline Total \\
\hline
\end{tabular}

\begin{tabular}{|r|r|}
\hline No. of Infected Animals & \\
\hline & 6 \\
\hline & 19 \\
56 \\
16 \\
\hline 3 \\
\hline $\mathbf{1 0 0}$ \\
\hline
\end{tabular}

Table.3 Breed wise occurrence of Malassezia dermatitis in dogs

\begin{tabular}{|l|}
\hline Breed \\
\hline Dalmatian \\
\hline Doberman Pinsher \\
\hline German Shepherd \\
\hline Great Dane \\
\hline Labrador Retriever \\
\hline ND \\
\hline Pug \\
\hline Spitz \\
\hline Total \\
\hline
\end{tabular}

\section{No. of Infected Animals}

\begin{tabular}{|r|r|}
\hline 2 \\
\hline 1 \\
11 \\
\hline 1 \\
13 \\
55 \\
\hline 4 \\
\hline 13 \\
\hline 100 \\
\hline
\end{tabular}

Table.4 Comparison of the Cytological Examinations

\begin{tabular}{|l|}
\hline Cytological \\
Examination \\
\hline Impression smear \\
\hline Adhesive tape \\
\hline Skin swab smear \\
\hline
\end{tabular}

\begin{tabular}{|l|}
$\begin{array}{l}\text { Cases positive } \\
(\%)\end{array}$ \\
\begin{tabular}{l}
26 \\
34 \\
\hline
\end{tabular} \\
\hline
\end{tabular}


Table.5 Frequency of $M$. pachydermatis isolation from different locations of dogs

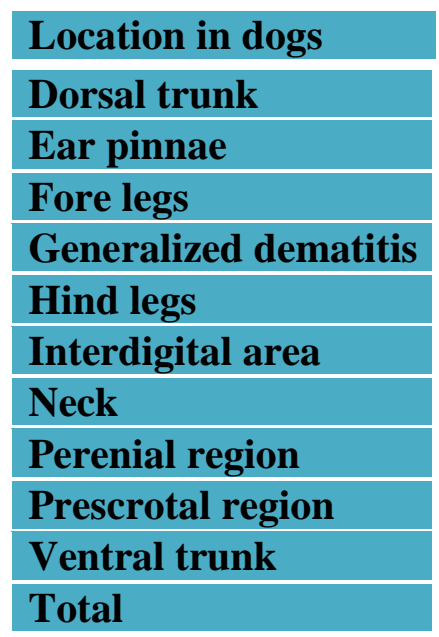

\section{No. of cultures obtained}

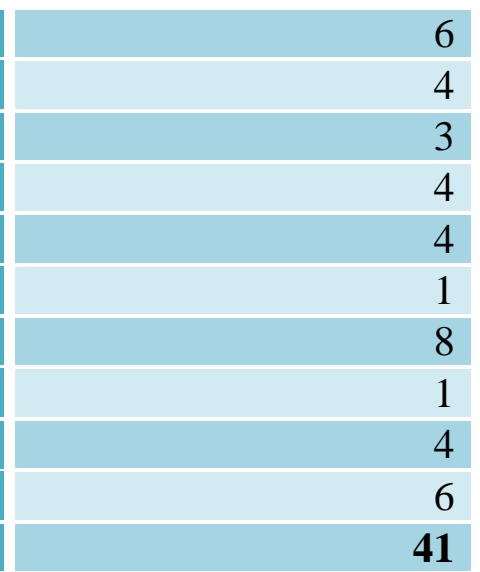

Table.6 Antifungal susceptibility of Malassezia pachydermatis isolated from Canine Dermatitis

\begin{tabular}{|l|r|r|}
\hline Antifungal drugs & Sensitive & Resistant \\
\hline Miconazole & 25 & 16 \\
\hline Ketoconazole & 41 & 0 \\
\hline Itraconazole & 41 & 0 \\
\hline Fluconazole & 41 & 0 \\
\hline Clotrimazole & 15 & 26 \\
\hline Nystatin & 0 & 41 \\
\hline Amphotericin B & 41 & 0 \\
\hline
\end{tabular}

Based on sex wise occurrence, highest incidence was observed in Male (69 nos) over female (31 nos) for Malassezia dermatitis. The chi-square analysis revealed that there is a significant difference between male and female dog's in the occurrence of Malassezia dermatitis $(\mathrm{P}<0.5)$. In contrary to this study no significant sex predilection for Malassezia infection was detected by Bond et al., (1996) and Nardoni et al., (2004).

The frequency of isolation was higher in neck region (8 nos) followed by dorsal and ventral trunk (6 nos), ear pinnae, hind legs, prescrotal region and in cases of generalized dermatitis (4 nos), fore legs (3 nos), perenial region and interdigital area $(1 \mathrm{no})$. The results of the present findings corroborates with the observations of Yurayart et al., (2010) who reported that highest number of isolates were obtained from the neck region followed by ear canal, interdigital area and groin.

\section{Cytological examination}

In impression smear examination, on an average 3-4 peanut or footprint shaped budding yeast cells per 1000X oil immersion field could be detected by Methylene blue staining. In adhesive tape smear examination, on an average 8-10 cells per field could be detected. Adhesive tape smear detected budding yeast cells more frequently when compared with direct impression smear examination. In skin swab smear examination, on an average 5-8 cells per field was detected. 
The results obtained by these three methods are depicted in Table 4. In the present study $26 \%$ cases were positive by impression smear, $34 \%$ cases were positive by adhesive tape and $40 \%$ cases were positive by skin swab smear. This study indicated that the impression smear, adhesive tape and the dry swab sampling techniques, successfully detected the yeast Malassezia on the skin of dogs. However, when the results of the impression smear, tape and swab techniques were compared; it was found that the swab technique detected the yeast on significantly more dogs than the impression smear examination and adhesive tape. The chisquare test analysis reveals that there is a significant difference between impression smear examination versus skin swab smear and adhesive tape techniques $(\mathrm{P}<0.0353)$. The chi-square analysis also revealed that there is no significant difference between skin swab smear and adhesive tape techniques $(\mathrm{P}>0.5)$. Omodo et al., (2003) reported that a swab is likely to be more practical than the adhesive tape when collecting material from the skin surface of a dog with a heavy greasy exudate as may be found in severe cases of seborrhoea. The exudate may prevent the tape from sticking to the glass and may also make it difficult to distinguish colonies of $M$. pachydermatis growing under the adhesive tape on culture. On the basis of the empirical evaluation of this study, skin swabs were considered to be the most reliable specimen because they may be used for both cytological examination and culture and are easy to use.

\section{Antifungal susceptibility test}

The antifungal susceptibility test was carried out with seven antifungal agents against 41 isolates of $M$. pachydermatis as per the standard disc diffusion method. All the isolates were susceptible to Amphotericin B, Ketoconazole, Itraconazole and Fluconazole followed by 25 isolates were susceptible to
Miconazole and 15 isolates were susceptible to Clotrimazole (Figure 5). A cent percent sensitivity recorded in this study indicates that any of the above drugs can be suggested for the treatment of $M$. pachydermatis dermatitis in canines. Moreover, all the 41 isolates were resistant to Nystatin followed by 26 isolates resistant to Clotrimazole and 16 isolates resistant to Miconazole. The study reveals that Nystatin should not be recommended for the treatment of $M$. pachydermatis dermatitis in canines. Variations in the antifungal sensitivity were observed only with Miconozole and Clotrimazole. Details of the antifugal susceptibility test of $M$. pachydermatis to seven antifungal drugs are presented in Table 5.

Clinical resistance to antifungal drugs by Malassezia species has only rarely been reported in veterinary or human medicine, and while the majority of studies have shown little evidence for in vitro antifungal resistance, multiple reports have demonstrated occasional very high anti-fungal MICs in individual Malassezia species and strains (Robson et al., 2010). Furthermore, resistance by $M$. pachydermatis has been shown to develop in vitro with multiple passage at near MIC concentrations of antifungals, suggesting that the cellular machinery exists in this species for development of possible clinically relevant resistance. More resistant species reported are $M$. pachydermatis (Nakamura et al., 2000), M. furfur (Duarte et al., 2006), M. globosa and M. restricta (Rincon et al., 2006). Whereas M. sympodialis has been frequently found more susceptible to the tested antifungals (Miranda et al., 2007). The susceptibility of the yeast isolates to antifungal agents via a disk diffusion methodology was assessed following disk diffusion guidelines and interpretive zone sizes based on the CLSI standard. Certain aspects of this guideline were modified to overcome problems associated with the 
colony characteristics, slower growth and incubation requirements of Malassezia species compared to Candida species in that: 1) Finding a suitable growth medium for Malassezia, especially the lipophilic species. This growth medium is often supplemented with a dispersing agent (mild detergent) to overcome the problem of cellular clumping which occurs due to the butyrous nature of this yeast. A dilute suspension of Tween 80 was added to the inoculum to act as a surfactant. 2) Increasing the inoculum size to counteract the slower growth rate of Malassezia compared to that of Candida species. The inoculum density was increased in order to produce semi-confluent growth. 3) The use of sterile glass beads and vigorous and continuous vortexing was essential to produce an even inoculum suspension. 4) Increasing the incubation time to up to 72 hours, again to counteract the slower growth rate of Malassezia compared to that of Candida species.

The present study revealed that the skin swabs were ideal clinical specimen for both cytological examination and isolation of Malassezia pachydermatis. Cultural studies show that SDA medium can be preferred over MDA for the isolation of Malassezia pachydermatis. Biochemical tests performed in isolates further confirmed for $M$. pachydermatis from the cases of canine dermatitis. Culture evidence indicated that only $41 \%$ of the cases were positive whereas $61 \%$ of cases were positive for Malassezia infection by PCR assay. Age-wise occurrence of Malassezia, the highest was noticed in one to three year old age dogs; Breed-wise occurrence, the highest was markedly prominent in Non-descriptive dogs and sexwise occurrence, the highest incidence observed in male dogs than females. The frequency of isolation was higher in neck region followed by other regions in the dogs. As per the antifungal susceptibility test performed; antifungal agents like Amphotericin B, Ketoconazole, Itraconazole and Fluconazole drugs can be suggested for the treatment of Malassezia dermatitis. Moreover, the study reveals that the drugs like Nystatin should not be recommended for the treatment of $M$. pachydermatis dermatitis in canines.

\section{References}

Ashbee, H.R and Evans, E.G. 2002. Immunology of diseases associated with Malassezia species, Clin. Microbiol. Rev. 15: $21-57$.

Bauer, A.W., Kirby, W.M.M., Sherris, J.C and Turck M. 1966. Antibiotic susceptibility testing by a standardized single disc method, Am. J. Clin. Pathol. 45: 493 - 496.

Bond, R., Ferguson, E.A., Curtis, C.F., Craig, J.M and Lloyd, D.H. 1996. Factors associated with elevated cutaneous Malassezia pachydermatis population in dogs with pruritic skin disease, J. Small Anim. Pract. 37: 103 - 107.

Cabanes, F.J., Theelen, B., Castellá, G and Boekhout, T. 2007. Two new lipiddependent Malassezia species from domestic animals, FEMS Yeast Res. 7: 1064 - 1076.

Cafarchia, C., Gasser, R.B., Luciana, A., Latrofa, M.S and Otranto. D. 2011. Advances in the identification of Malassezia, Mol. Cellular. Probes. 30: $1-7$.

Cannon, P.F. 1986. International Commision on the Taxonomy of Fungi (ICTF). Name changes in fungi of microbiological, industrial and medical importance, Part 1, Microbiol. Sci. 3: $168-171$.

Chang, H.J., Miller, H.L and Watkins, N. 1998. An epidemic of Malassezia pachydermatis in an intensive care nursery associated with colonization of 
health care workers' petdogs, New England, J. Med. 338: 706 - 711.

Charach, M. 1997. Can. Vet. J. 38: 311.

Chen, T.A and Hill, P.B. 2005. The biology of Malassezia organisms and their ability to induce immune responses and skin disease, Vet. Dermatol. 16: 4.

Duarte, R.J and Hamdan, J.S. 2006. Susceptibility of yeast isolates from cattle with otitis to aqeous solution of povidine iodine and to alcohol ether solution, Med. Mycol. 44(4): 369 - 373.

Galuppi, R., Bonoli, C., Guardini, G., Ostanello, F and Tampieri, M.P. 2010. Variability of Malassezia pachydermatis an Italian experience and brief review of literature, Mikologia. Lekarska. 17: 87 - 96.

Girao, M.D., Prado, M.R., Brilhante, R.S.N., Cordeiro, R.A., Monteiro, A.J., Sidrim, J.J.C. and Rocha, M.F.G. 2006. Malassezia pachydermatis isolated from normal and diseased external ear canals in dogs: A comparative analysis, $J$. Clin. Microbiol. 172: 544 - 548.

Gueho, E., Midgley, G and Guillot, J. 1996. The genus Malassezia with description of four new species, Antonie van Leeuwenhoek. 69: 337 - 355.

Guillot, J and Gueho, E. 1995. The diversity of Malassezia yeasts confirmed by rRNA sequence and nuclear DNA comparisons, Antonie Van Leeuwenhoek. 67: 297 - 314.

Hirai, A., Kano, R., Makimura, k., Duarte, E.R., Hamdan, J.S and Lacahance. 2004. Malassezia nana sp. Nov, a novel lipid dependent yeast species isolated from animals, Int. J. Syst. Evol. Microbiol. 54: 623 - 627 .

Kaneko, T., Makimura, K., Sugita, T and Yamaguchi, H. 2006. Tween 40-based precipitate production observed on modified chromogenic agar and development of biological identification kit for Malassezia species, Med. Mycol. 44: $227-231$.

Keddie, F.M. 1966. Electron microscopy of Malassezia furfur in tinea versicolor, Sabouraudia. 5: 134 - 137.

Larson, C.E., Larson, M.H.M.A., Amaral., Gandra, C.R.P., Hagiwara, M.K and Fernandes. 1988. Dermatitis in dogs caused, by Malassezia (Pilyrosporum) pachydermatis, Arsveterinaria. 4: 63 68.

Mansfield, P.D., Boosinger, T.R and Attleberger, M.H. 1990. Infectivity of Malassezia pachydermatis in the external ear canal of dogs, J. Am. Anim. Hosp. Assoc. 26: 97 - 100.

Mason, K.V and Evans, A.G. 1991. Dermatitis associated with Malessezia pachydermatis in $11 \mathrm{dogs}, J$. Am. Anim. Hosp. Assoc. 27: 13 - 20.

Maulldin, E.A., Scott, D.W., Miller, W.H. Jr and Smith, C.A. 1997. Vet. Dermatol. 8: 191. Mayser, P., Haze, P and Papavassilis, C. 1997. Differentiation of Malassezia species: selectivity of Cremophor EL, castor oil and ricinoleic acid for M. furfur, Br. J. Dermatol. 137: $208-213$.

Miranda, K.C., Rodrigues, C., Passos, X.S., Fernandes, O.F and Maria do Rosario Rodrigues, M.R. 2007. Antifungal activities of azole agents against the Malassezia species, International J. of Antimicrobial Agents; 29: 281 - 284.

Muller, G.M., Kirik, R.W and Scott, D.W. 2001. Malassezia dermatitis, otitis externa, Small. Ani. Dermatol. 6th editi. p: $364-375$.

Nakamura, Y., Kano, R., Marai, T., Watanabe, S and Hasegawa, A. 2000. Susceptibility Testing of Malassezia Species Using the Urea Broth Microdilution Method, Antimicrob. Agents. Chemther. 2185 - 2186.

Nardoni, C., Dini, M., Taccini, F and Mancianti, F. 2006. Occurrence, 
distribution and population size of Malassezia pachydermatis on skin and mucosae of atopic dogs, Vet. Microbiol; 122: $172-177$.

Nardoni, S., Corazza, $\mathrm{M}$ and Mancianti, F. 2008. Diagnostic and clinical features of animal Malasseziosis, Parasittol. 4: 227 -229 .

Nardoni, S., Mancianti, F., Rum, A and Corazza, M. 2004. Isolation of Malassezia species from healthy cats and cats with otitis, J. Feline. Med and Surgery. 7: $141-145$.

Nicklas, W and Mumme, J. 1979. Mykologische and bakteriologische Untersuchungenzur Keimflorabei der Otitis externa des Hundes, Tierärztliche Umschau. 34: 606 - 615.

Omodo, E., Baker, K.P and Fuller, H. 2003. Comparison of two sampling techniques for the detection of Malassezia pachydermatis on the skin of dogs with chronic dermatitis, The Vet. J. 165: 119 $-124$.

Plant, J.D., Rosenkrantz, W.S and Griffin, C.E. 1992. Factors associated with and prevalence of high prevalence of high Malassezia pachydermatis numbers on dog skin, J. American. Vet. Med. Assoc. 201: $879-882$.

Rincon, S., Cepero de garci, M.C and Espinel ingroff, A. 2006. A modified Christensen's urea and CLSI broth microdilution method for testing susceptibilities of six Malassezia species to voriconazole, itraconazole and ketoconazole, J. Clin. Micro. 44(9): $3429-3431$.
Robson, D., Moss, S., Trott, D., Burton, G and Basett, R. 2010. Evidence for possible clinically relevant antifungal resistance in Malassezia pachydermatis: 10 cases, Dermatology chapter of the AVCSc Science Week Proceedings. 2 3 July.

Scott, D.W. 1992. Bacteria and yeast on the surface and within non inflamed hair follicles of skin biopsies from dogs with non-neoplastic dermatoses, The cornell Vet. 82: 371-377.

Sugita, T., Tajima, M., Takashima, M., Amaya, M., Saito, $\mathrm{M}$ and Tsuboi, R. 2004.A new yeast, Malassezia yamatoensis, isolated from a patient with seborrhoeic dermatitis, and its distribution in patients and healthy subjects, Microbial immunol. 48: 579 583.

Yurayart, C., Chindamporn, A., Suradhat, S., Tummaruk, P., Kajiwara, S. and Prapasarakul, N. 2010. Comparative analysis of the frequency, distribution and population sizes of yeasts associated with canine seborrheic dermatitis and healthy skin, Vet. Microbiol. 148: 356 - 362.

Zhang, K., Sparling, J., Chow, B.L., Elsayed, S., Hussain, Z., Chruch, D.L., Gregson, D.B., Louie, T and Conly, J.M. 2004. New Quadriplex PCR assay for detection of methicillin and mupirocin resistance and simultaneous discrimination of S.aureus from coagulase-negative Staphylococci, $J$. Clin. Microbiol. 42: 4947 - 4955.

\section{How to cite this article:}

Uday Seetha, Sumanth Kumar, Raghavan Madhusoodanan Pillai, Mouttou Vivek Srinivas, Prabhakar Xavier Antony and Hirak Kumar Mukhopadhyay. 2018. Malassezia Species Associated With Dermatitis in Dogs and Their Antifungal Susceptibility. Int.J.Curr.Microbiol.App.Sci. 7(06): 1994-2007. doi: https://doi.org/10.20546/ijcmas.2018.706.236 